\title{
Polycystic kidney disease prevented by transgenic RNA interference
}

\author{
Cell Death and Differentiation (2005) 12, 831-833. doi:10.1038/sj.cdd.4401603 \\ Published online 1 April 2005
}

Dear Editor,

There is currently substantial hope that RNA interference (RNAi)mediated modulation of protein expression can be used for prevention or treatment of diseases, ${ }^{1}$ but only a few studies in whole animals have been reported so far. Here, we show that transgenic expression of a short hairpin (sh) RNA targeting Bim efficiently prevents the development of polycystic kidney disease in Bcl-2-deficient mice and that this effect can be transmitted over several generations. This is the first time that a disease due to a genetic defect has been corrected by using transgenic RNAi.

Mice lacking the antiapoptotic protein $\mathrm{Bcl}-2$ invariably develop fatal polycystic kidney disease associated with growth retardation. ${ }^{2} \mathrm{Bcl}-2$-deficient mice are also lymphopenic and their coat turns grey due to degeneration of melanocytes. ${ }^{2}$ We have previously demonstrated that ablating the proapoptotic gene $\mathrm{Bim}$ from $\mathrm{BCl}-2^{-1-}$ mice prevents the development of all degenerative disorders present in these mice. ${ }^{3}$ Removal of a single allele of Bim was even sufficient to prevent polycystic kidney disease in these mice. This effect is specific to Bim, since ablating Bax or Blk in the $B \mathrm{Cl}-2^{-1-}$ mice had no effect on the polycystic kidney disease phenotype..$^{4,5}$ Since the phenotypic abnormalities of $\mathrm{Bcl}-2^{-1-}$ mice are exquisitely sensitive to the dosage of Bim, we used this mouse model to evaluate the effectiveness of RNAi in vivo and its potential as a therapeutic tool.

Small interfering RNAs (siRNAs) and shRNAs promote the degradation of messenger RNA in the process of RNAi. ${ }^{6,7} \mathrm{We}$ generated 18 anti-Bim shRNA constructs in the pSuper vector as described. ${ }^{8}$ The ability of each construct to reduce Bim protein expression was tested by cotransfection with the reporter pEF-FLAG-BimEL $\triangle \mathrm{BH} 3$ (the $\mathrm{BH} 3$ region was removed to prevent killing of transfected cells, thereby maximising protein production) in Cos cells, and assessing the amount of Bim protein expressed from the reporter after $36 \mathrm{~h}$ by anti-FLAG mAb Western blotting. Only one construct, termed pSuper-bim73, reduced the expression of Bim significantly (Figure 1a). It contained the sequence $5^{\prime}$ TGATGTAAGTTCTGAGTGTG-3', which is common to all known Bim mRNA isoforms and is also $100 \%$ conserved in human BIM. A control construct, termed pSuper-bim75, was produced by introducing a single mismatch in the siRNA sequence (5'-TGATGTAAGGTCTGAGTGTG-3'). It did not interfere with the expression of Bim in the cotransfection experiments in Cos cells (Figure 1a).

To test the long-term activity of these shRNAs, we added a PGK-Hygro-PolyA cassette to each construct and generated stable transfectants of MCF-7 cells, which normally express relatively high levels of Bim protein. The cells were maintained in culture for several weeks before assessing their levels of Bim expression. pSuper-bim73 appeared to downregulate Bim expression in MCF7 cells as well as in the transient transfection system. In contrast to the results obtained by short-term cotransfection, however, MCF-7 cells expressing shRNA-bim75 also had much reduced levels of Bim protein (Supplementary Figure S1 online). It is likely that the prolonged expression of the mismatched shRNA and levels of RNA expression lower than those obtained upon transfection are responsible for this difference.

For the experiments within the whole animal, a transgene was generated by cloning a pEF-neo-PolyA cassette into pSuper-bim73, with its transcription orientation opposite to that of the H1-Bim-siRNA. This construct was electroporated into the C57BL/6 embryonic stem cell line Bruce4, and transfected cell clones were selected for their resistance to G418. Since Bim protein can be detected in mouse ES cell lysates, we prepared lysates from 80 G418-resistant clones and determined their Bim content by Western blotting (Figure 1b). About $10 \%$ of G418-resistant clones had reduced levels of Bim. We selected three clones showing a significantly reduced Bim expression (6F1, 5A1 and 2D2). These clones were shown to be independent by Southern blotting with a neo probe (Figure 1c). Clones 6F1 and 2D2 appear to have a single site of integration of the transgene, whereas 5A1 appears to have two different sites and several copies of the transgene in one of these sites. Since downregulation of Bim was similar for all three clones, we chose clones 6F1 and 2D2 to engineer transgenic mice by injection of the ES cells into blastocysts, and breeding of the resulting chimeric mice with selection for germline transmission. Two independent strains of transgenic animals, 434 and 436, were generated from clones $6 \mathrm{~F} 1$ and 2D2, respectively. In both strains, Bim proteins were still readily detectable (in the organs tested), but at clearly reduced levels relative to those of wild-type mice (Figure 1d, and data not shown). The downregulation of Bim expression was stable over several generations, as Bim mRNA levels were reduced in all tissues examined in transgenic mice of the fourth generation (Figure 1e and data not shown).

RNAi-transgenic $(\mathrm{Tg})$ animals (strain 434) were crossed twice with $\mathrm{BCl}-2^{+/-}$mice to generate $\mathrm{Bcl}-2^{-/} / \mathrm{Tg}$ mice. In contrast to $\mathrm{Bcl}-2^{-1-}$ mice, $\mathrm{Bcl}-2^{-/-} / \mathrm{Tg}$ mice did not show any sign of growth retardation, and became healthy adults (Figure 


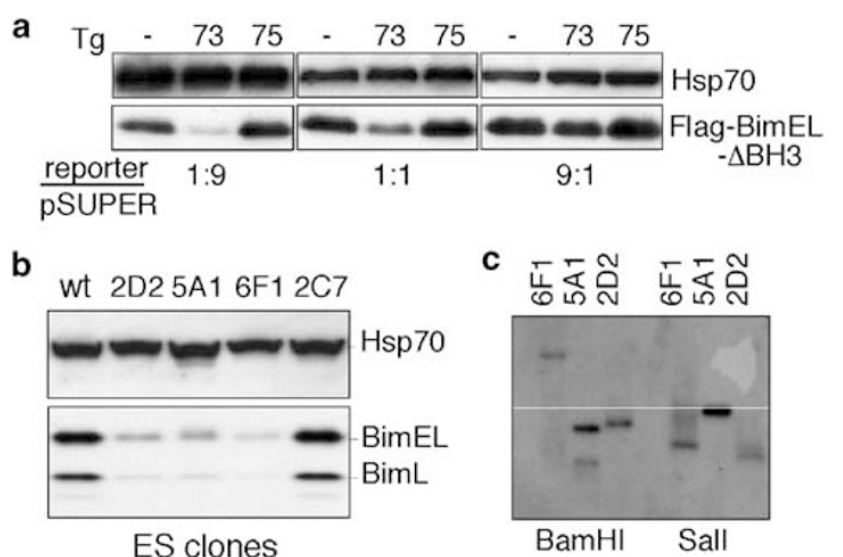

d Bim protein

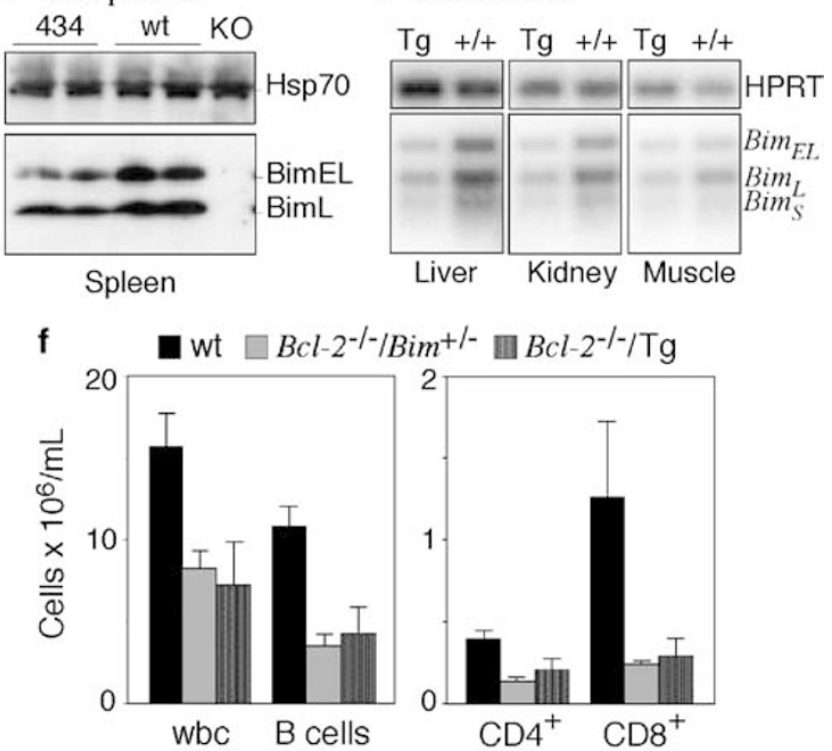

Figure 1 (a) pSuper-bim73 reduces expression of pEF-FLAG-BimEL $\triangle B H 3$ protein in cotransfection experiments. The interference efficiency of the RNAi constructs was assayed by cotransfection in Cos cells. (b) siRNA73 knocks down Bim expression in C57BL/6 Bruce4 embryonic stem cells. (c) Southern blot with a neo probe shows that clones $2 \mathrm{D} 2,5 \mathrm{~A} 1$ and $6 \mathrm{~F} 1$ are independent. Clones $6 \mathrm{~F} 1$ and 2D2 were used to generate transgenic mouse lines 434 and 436 , respectively. (d) Reduced levels of Bim in RNAi-transgenic mice (434) compared to wild-type (wt) mice are shown. Lysates of $2 \times 10^{6}$ spleen cells were assayed for their content of Bim and Hsp70. A Bim ${ }^{-l-}$ mouse was used as a control (KO). (e) Bim mRNA levels were reduced in all tissues examined in fourth generation transgenic mice as assessed by RT-PCR. (f) Lymphocyte numbers are similar in adult $\mathrm{BCl}-2^{-l-} / \mathrm{Tg}$ and $\mathrm{BCl}-2^{-l-} / \mathrm{Bim}^{+1-}$ mice. $\mathrm{BCl}-2^{-1-}$ mice could not be included in this study because all die early from polycystic kidney disease. We have demonstrated previously by haemopoietic stem cell reconstitution experiments that lymphopenia of $B \mathrm{Cl}-2^{-l-}$ mice is partially rescued in $B \mathrm{Cl}-2^{-l-}$, $\mathrm{Bim}^{+/-}$mice (2)

2a and b). None developed polycystic kidneys (Figure 2c), further confirming the stability of the shRNA expression over four generations. Consistent with the fact that Bim expression was reduced, but not ablated by the shRNA, $\mathrm{BCl}-2^{-1-} / \mathrm{Tg}$ mice still turned grey at the second hair follicle cycle (Figure 2b). Apoptosis in the spleen was greatly reduced compared to that observed in $\mathrm{BCl}_{-2}^{-/-}$mice (Figure 2d).
$B C l-2^{-1-}$ mice display a very small thymus and spleen, and the presence of many apoptotic cell corpses denote intense apoptosis in these organs. Spleens and thymi of $\mathrm{BCl}^{-2} 2^{-1} / \mathrm{Tg}$ mice were five to 10 times bigger than those of $\mathrm{BCl}^{-2^{-/-}}$mice, and the presence of cell corpses in these tissues was considerably reduced (Figure $2 \mathrm{~d}$, and data not shown). The lymphopenia was partially rescued in $\mathrm{BCl}-2^{-1-} / \mathrm{Tg}$ mice, as their leucocyte numbers were similar to those found in $\mathrm{BCl}_{-2} \mathrm{2}^{-/} / \mathrm{Bim}^{+-}$mice $^{3}$ (Figure 1f). In all aspects, $B \mathrm{Cl}-2^{-1-} / \mathrm{Tg}$ mice appear almost identical to the $\mathrm{BCl}_{-2}^{-1-} /$ $\mathrm{Bim}^{+/-}$mice.

Examination of the numbers of transgenic mice (without the $b c l-2$-null mutation) in our colonies after weaning and genotyping revealed a $60 \%$ loss of transgenic mice in both strains 434 and 436 . Closer examination showed that an unusually high number of mice died suddenly in the third week of life, just prior to weaning. This result was surprising since neither $\mathrm{Bim}^{+/-}$nor $\mathrm{Bim}^{-/-}$mice die at such an early age. Despite a thorough histological examination, and having dismissed the most likely candidate reasons for sudden death (haemorrhage, heart failure, pulmonary embolism), we could not find a histological clue to explain the sudden death of all these animals. It is possible that the mice succumbed to cardiac arrhythmia, as this disease causes sudden death without leaving a histological signature.

The use of RNAi as a therapeutic tool has long been predicted and a few studies in mouse have been reported that showed that it may be used to downregulate expression of viral $^{9}$ or cellular genes ${ }^{10,11}$ in vivo. Our results demonstrate for the first time that the expression of a transgenic shRNA can prevent the development of a disease that is caused by a genetic defect. Moreover, the shRNA expression had to be maintained for four generations before its effects on the development of polycystic kidney disease in $\mathrm{BCl}^{-2^{-1-}}$ mice could be observed. We may imagine that an anti-Bim shRNA transgene in bone marrow stem cells could confer a survival advantage upon them and hence improve the haematopoietic system repopulation after bone marrow transplantation. Although the use of a transgene may not be practicable in humans, it could readily be envisioned in livestock to produce animals resistant to certain viruses or other pathogens. The death of a significant proportion of the transgenic animals in the present study is a major source of concern with regard to the use of the strategy described here. It is not clear whether this will turn out to be a general problem or is linked to the sequence of the particular shRNA against Bim. Although a BLAST search with that sequence in many of the available databases failed to pick up any perfect match in the rest of the mouse genome or expressed sequence tags, we cannot exclude that the death of these animals may be the consequence of an off-target effect on an unidentified gene. It has been reported that RNAi may be far less specific than initially believed, and that the off-target effect of siRNAs may be triggered by homology stretches of 11 nucleotides or more. ${ }^{12,13}$ Since none of the 17 other constructs against Bim that we generated in pSuper worked in the transient transfection assay, we could not use them to generate other animals to study this effect further. This result, however, should remind us that caution is necessary when analysing the results of RNAi experiments. 

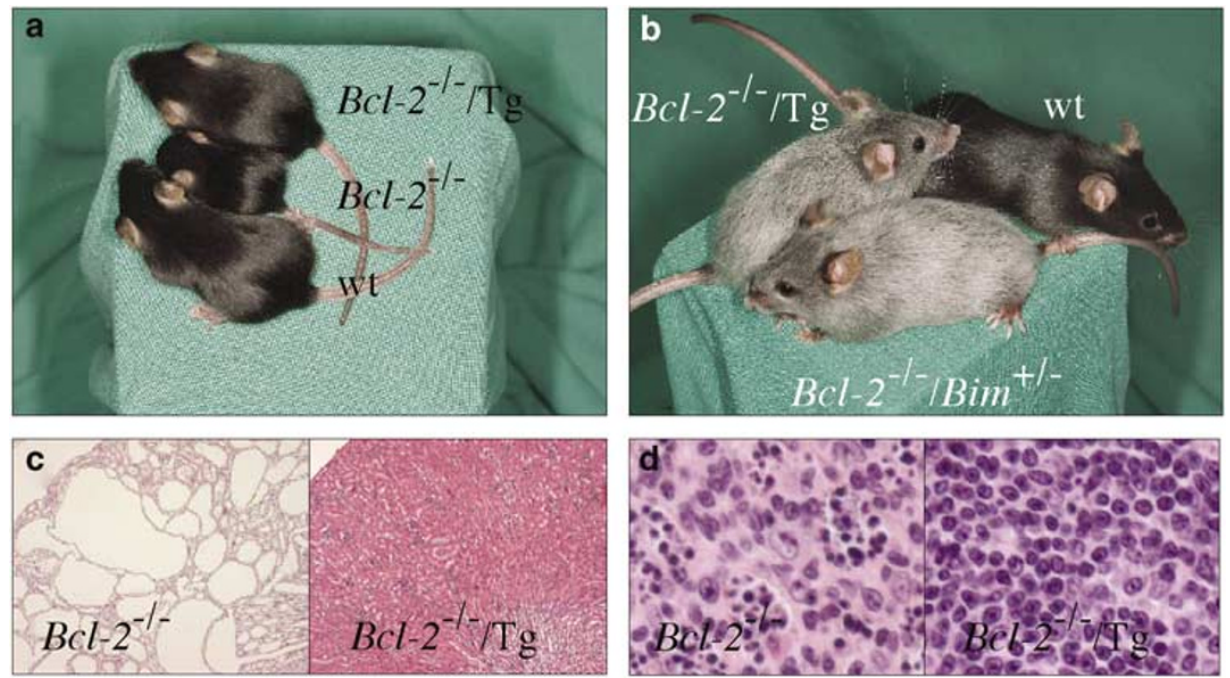

Figure 2 A transgenic anti-Bim siRNA prevents polycystic kidney disease in $B \mathrm{Cl}-2^{-1-}$ mice. (a) $B \mathrm{Cl}-2^{-1-} / \mathrm{Tg}$ mice are not runted (here at 3 weeks of age). (b) $B \mathrm{Cl}-2^{-1-}$, $\mathrm{Tg}$ mice grow to healthy adults (here at 10 weeks of age), but they still turn grey as do the $B \mathrm{Cl}_{-2} \mathrm{-}^{-1-} / \mathrm{Bim}^{+1-}$ mice. (c) Kidneys of $B \mathrm{Cl}-2^{-1-} / \mathrm{Tg}$ mice are not polycystic ( $\times 20)$. (d) Reduced apoptosis in the spleen of $\mathrm{Bcl}^{-2}{ }^{-1-} / \mathrm{Tg}$ mice $(\times 50)$ is shown

\section{Acknowledgements}

We thank Drs. JM Adams and AW Harris for critical reading of the manuscript, and Dr. R Agami for the pSuper vector. This work was supported by fellowships and grants from the $\mathrm{NCl}$ (CA43540 and CA80188), the NHMRC, the Leukemia and Lymphoma Society (LLS), and the Sylvia and Charles Viertel Charitable Foundation.

\section{$P$ Bouillet ${ }^{*, 1}$, M Robati $^{1}, M$ Bath $^{1}$ and A Strasser $^{1}$}

1 The Walter and Eliza Hall Institute of Medical Research, Melbourne, Australia

* Corresponding author: P Bouillet, Molecular Genetics of Cancer Division, The Walter and Eliza Hall Institute of Medical Research, Melbourne, 1G Royal Parade, Parkville, Australia. Tel: +61 39345 2334; Fax: + 6139347 0852; E-mail: bouillet@wehi.edu.au
1. Dykxhoorn DM et al. (2003) Nat. Rev. Mol. Cell. Biol. 4: 457467

2. Veis DJ et al. (1993) Cell 75: 229-240

3. Bouillet $P$ et al. (2001) Dev. Cell 1: 645-653

4. Knudson CM and Korsmeyer SJ (1997) Nat. Genet. 16: 358-363

5. Coultas L et al. (2004) Mol. Cell. Biol. 24: 1570-1581

6. Elbashir SM et al. (2002) Nature 411: 494-498

7. McManus MT and Sharp PA (2002) Nat. Rev. Genet. 3: 737-747

8. Brummelkamp TR et al. (2002) Science 296: 550-553

9. Giladi $\mathrm{H}$ et al. (2003) Mol. Ther. 8: 769-776

10. Song $E$ et al. (2003) Nat. Med. 9: 347-351

11. Tiscornia G et al. (2003) Proc. Natl. Acad. Sci. USA 100: 18441848

12. Jackson AL et al. (2004) Nat. Biotech. 21: 635-637

13. Scacheri PC et al. (2004) Proc. Natl. Acad. Sci. USA 101: 18921897

Supplementary Information accompanies the paper on Cell Death and Differentiation website (http://www.nature.com/cdd) 